\title{
La educación a distancia, reduce las distancias
}

Fecha de recepción: 11 de junio de 2009

Fecha de aprobación: 1 junio de 2010

\section{César Augusto Sierra Varón}

csierrav@poligran.edu.co

Politécnico Grancolombiano

\begin{abstract}
Reseña del autor
Psicólogo de la Universidad Javeriana de Bogotá, con conocimiento y experiencia práctica en áreas como psicología clínica y educativa. Docente a cargo de las cátedras Desarrollo, Clínica infantil, Planeación y ejecución de talleres -conferencias para padres de niños y adolescentes-, impartidas por el programa de Psicología, que pertenece a la Facultad de Ciencias Sociales del Politécnico Grancolombiano. Psicoterapeuta de niños y adolescentes. Asesor pedagógico en la autoría de textos de educación preescolar de la Editorial Creativa.
\end{abstract}

\section{Resumen}

Este ensayo describe algunos aspectos relevantes de la modalidad de educación a distancia, y expone cómo ésta reduce las distancias físicas para acceder a sistemas de educación superior. El autor trabaja desde su propia experiencia como estudiante de especialización a distancia, comparada con el rol de docente universitario en educación presencial. Así, expone algunas ventajas de la modalidad de educación a distancia, frente a la educación presencial.

La educación brinda una herramienta que contribuye al desarrollo de la autonomía en los procesos de aprendizaje de los estudiantes, siempre y cuando algunos factores internos -como la motivación y la comprensión lectoraapoyen dichos procesos. Estos factores son fundamentales para el desarrollo posterior de la capacidad de aprender a aprender.

\begin{abstract}
This essay describes some relevant aspects of remote education modality, and shows how this one reduces physical distances, in order to access to superior education systems. The author works from his own experience as a specialization at distance student compared with his roll as a college teacher in regular education. In this way, he exposes some advantages of remote education modality faced with regular education.

Education offers a tool that contributes to the development of the autonomy in the students' learning processes, if some internal factors -such as motivation and reading comprehension-support those processes. Those factors are fundamental to later develop of the ability of learn to learn.

The remote education allows achieve great goals, avoiding the distance as an excuse,
\end{abstract}


La educación a distancia permite alcanzar grandes objetivos, si se deja de lado la excusa de la distancia, ya que esta modalidad lleva la educación a nuestros hogares, en lugar de que nosotros tengamos que desplazarnos a los establecimientos educativos.

Palabras clave

Educación a distancia, autonomía, aprendizaje. because this modality brings the education to our homes, instead of we must move to the educative establishments.

\section{Keywords}

Remote Education, Autonomy, Learning.

La educación a distancia brinda posibilidades para adquirir y generar conocimientos, desarrollar habilidades y destrezas a lo largo de la vida de la persona; tiene en cuenta su estado actual educativo

y cultural, y la ubica en el desarrollo bistórico del conocimiento teniendo en cuenta sus valores sociales y culturales para que, una vez los apropie, genere actitudes creativas frente a ellos, por el concepto de educación permanente...

(Ferroni et al., 2005)

Muchos aspectos de la humanidad sobre su propia evolución van cambiando, van mejorando, se van transformando. ¿Para qué? Se esperaría que todo cambio que implique evolución -y no involución- conllevara a mejorar la calidad de vida de las personas; tales aspectos tienen que ver con el progreso de todas las condiciones que el medio le ofrece.

A medida que el tiempo pasa, la educación se instaura como uno de los aspectos más importantes en la formación de cualquier persona. La educación, entonces, también queda inscrita en ese proceso de cambio, que va de la mano con el desarrollo tecnológico que la ciencia nos brinda. Por eso, este artículo abordará lo que se percibe sobre la evolución de la educación, hasta llegar en la actualidad a la alternativa de la educación a distancia, mencionando los factores que influyen para que ésta sea exitosa.

Hemos recurrido a diversas herramientas para educar a nuestros semejantes: la tradición oral, que consiste en transmitir nuestro legado cultural por medio de los relatos de los sabios de cada cultura; el descubrimiento de un lenguaje pictórico, mediante el que se puede plasmar formas de ser, tradiciones, conocimientos 
y enseñanzas; y el lenguaje escrito, con el que el hombre ha podido enseñar y educar de diferentes formas a los integrantes de su propio grupo cultural.

A partir de la invención de la imprenta, ya no era necesario escribir y escribir, encerrado durante largas horas, para plasmar ideas en un material que se pudiera leer, y así transmitir ese conocimiento. La siguiente etapa de este proceso es la aparición de las primeras ediciones de textos en varias lenguas, lo que amplió el alcance del conocimiento lo trasladó a largas distancias.

Con la pedagogía, fundamentada en este gran avance, las escuelas transmiten los conocimientos basándose en la importancia de la educación y la alfabetización. Entonces se recurrió a medios como la tablilla, el tablero de tiza, los cuadernos, el tablero acrílico con sus respectivos marcadores borrables de colores vivos. Poco a poco, estos elementos se han desdibujado, gracias a los nuevos desarrollos tecnológicos en el campo de la información y la comunicación. La presencia de los computadores desmontó los espacios y los objetos físicos, para implementar los espacios y los objetos virtuales.

Ya no es necesario escribir sobre papel para leer, ya no necesitamos trasladarnos a salones y espacios físicos para educarnos, formarnos o recibir información. Los espacios virtuales han transformado las modalidades pedagógicas anteriores, que se basaban en la educación mediante la modalidad presencial como única opción, es decir, el estudiante debía asistir a un salón o a algún sitio para esperar la presencia de algún experto en cualquier tema, para que simplemente tuviéramos la oportunidad de escucharlo y aprender así, como si sólo fuéramos receptores de conocimiento.

Los tiempos cambian, la forma de educación, también. Ya no es necesario asistir a espacios físicos, sino que es posible acceder a estudios de formación académica desde cualquier lugar, por medio de la educación a distancia, que ayuda a que las distancias ya no sean un impedimento para acceder a diferentes niveles de formación académica. Este tipo de educación disminuye las distancias, porque permite recibir formación académica a kilómetros de las instalaciones de un centro educativo.

A pesar de que los tiempos y el sistema cambien, el objetivo es el mismo: educar y formar a otra persona, de ahí la importancia del estudiante en el proceso educativo -ya sea presencial o a distancia-. Rosalba Rodríguez (2009) sustenta esta idea en su documento Metodología del trabajo académico, al afirmar que "históricamente el centro de la educación a distancia lo ocupa el estudiante, sujeto activo y responsable, capaz de encargarse autónomamente de su propio aprendizaje, empleando para ello diversas estrategias, desarrolladas con el apoyo de diferentes mediaciones y medios pedagógicos" (Rodríguez, 2009). 
Esto conlleva a la implementación de las tics -Tecnologías de la Información y la Comunicación-, un factor de gran influencia en la educación a distancia ya que, con la llegada de las nuevas tecnologías, la educación se configura como una alternativa para muchos países. Las TICs apoyan la modalidad de educación a distancia pues, gracias a ellas, se conserva el aspecto esencial en una modalidad de educación en lo que la interacción presencial directa entre el docente y el alumno no es necesaria.

Sin embargo, recibir educación a distancia no implica que ya no exista comunicación entre docente -tutores- y alumnos, sino que dicha comunicación no presencial se lleva a cabo a través de los medios de comunicación. Así, la educación a distancia ofrece una manera de aprendizaje diferente, en la que el estudiante debe desarrollar su autonomía, autorregulación, disciplina y hábitos de estudio con mayor énfasis, ya que él mismo se encarga de obtener el mayor provecho de su proceso de formación académica (Ferroni y Velásquez, 2005). Educación a distancia no significa aislamiento total, sino que constituye una modalidad con la que el estudiante fortalece la autonomía en su proceso de aprendizaje.

La educación a distancia y las nuevas Tics van de la mano. Rodríguez, Hernández y Albarracín (2008) plantean algunas ventajas de estas herramientas:
- Facilitan la comunicación entre profesores y alumnos, eludiendo los problemas de horarios y distancias.

- Crean nuevos canales de comunicación entre los estudiantes, según sus intereses e inquietudes.

- Suministran una cantidad de información enorme, con gran rapidez y a un bajo costo.

La distancia se convierte en un concepto relativo -aunque todavía se considera muy poco-, ya que la perspectiva de distancia depende desde dónde se mire. Por ejemplo, la distancia entre personas supone algún problema entre ellas, aunque sea pequeño o involuntario; la distancia entre países se asocia con negociaciones largas e interminables; la distancia entre ideas significa un esfuerzo para encontrar el consenso entre ellas. Aunque la distancia también significa respeto, consideración, poder, atención y hasta admiración del maestro al discípulo, del jefe al subordinado o, simplemente, del hermano mayor al más pequeño.

Pese a esto, la educación a distancia no cuenta con la valoración y respeto que merece, es más, padece de cierto desprestigio; aunque también es altamente valorada por otros colectivos y por el contexto social en el que vivimos (Badia y Monimó, 2001).

Volvamos a nuestra tesis: la educación a distancia disminuye las distancias. Ferroni y Velázquez (2005) evidencian 
esta premisa al proponer varios rasgos principales de la educación a distancia:

- Está dirigida a una población estudiantil relativamente dispersa y en su mayoría adulta.

- Cuenta con una orientación autoinstruccional.

- Sus cursos son diseñados con una predominancia de textos impresos, que se transmiten por otros medios.

- Las comunicaciones son masivas.

- La comunicación es bidireccional.

- Sus estructuras curriculares son flexibles, organizadas en módulos y créditos.

La educación a distancia no puede ofrecer sus ventajas por sí sola, ya que depende de ciertos factores, como la motivación de los estudiantes, que influyen en el éxito de esta experiencia de formación autónoma.

La motivación es un proceso psicológico que permite cumplir propósitos con una actitud activa frente a los retos que se nos presenten. Es muy importante en el proceso de educación a distancia, en especial en pre y posgrado, ya que el estudiante sólo cuenta con su propia disciplina y autonomía para cumplir los objetivos. En esta modalidad no debe cumplir horarios específicos, no hay profesores que, le pidan a los estudiantes que cumplan con sus actividades, tampoco se cuenta con compañeros que lo distraigan. La motivación, entonces, se inscribe como uno de los pilares de la educación a distancia.

La educación presencial es sensible a factores externos, que el estudiante no puede controlar; en cambio, la modalidad a distancia se caracteriza por presentar factores internos, como la motivación, que ayudarán al estudiante a dar cuenta de su proceso. Debido al carácter intrínseco al individuo, sí es posible ejercer control personal sobre este factor, pues a "la motivación que [se] origina en el mismo sujeto se le denomina motivación intrínseca; ésta está casi siempre relacionada con el deseo personal de satisfacer necesidades, intereses y expectativas y cuyos resultados se esperan disfrutar..." (Rodríguez, 2009).

La motivación brindará satisfacción personal a los estudiantes de educación a distancia, y encaminará el desarrollo de actividades propuestas para tal metodología. Sin embargo, si no se cuenta con una motivación interior óptima para continuar con el proceso de educación a distancia, es muy fácil fracasar en este intento, ya que no se percibirá utilidad y, por lo tanto, es muy probable que el estudiante deserte.

Por desgracia, quienes no cuentan con una motivación interna plena, sólo funcionan en sus actividades y en su vida personal bajo satisfacciones externas a ellas -presiones sociales, satisfacción de otras personas, metas difusas-, lo que conduce a que estas personas 
tengan muy pocas satisfacciones por sus logros personales.

En el marco de la educación a distancia, encontramos otro factor de vital importancia y muy influyente en este proceso, dicho factor es la autonomía, que hace referencia al momento en el que el estudiante toma conciencia de que él sólo debe asumir su proceso de formación académica, con orientación, claro está.

La autonomía juega un papel fundamental en el contexto de la educación y la formación de las personas ya que, como lo mencionan Ferroni y Velásquez (2005), el aprendizaje es un proceso autónomo en el que el estudiante se acerca a los conocimientos teóricos y a su aplicación, a partir de la toma de conciencia sobre su responsabilidad sobre cuándo, cómo y cuánto debe aprender, para aprovechar un área o un quehacer específico.

El estudiante aprende de manera autónoma cuando comprende los contenidos, los textos, los conceptos, las actividades, sin que medie la presencia física de su tutor. Este proceso posibilita la realización personal del proyecto de vida, porque contribuye a adquirir las habilidades propias para continuar con el proceso de aprendizaje por cuenta propia: desarrolla autodisciplina, autogestión, compromiso personal, aspectos que, vistos en el contexto educativo, ayudarán a formar a las personas a lo largo su vida; tal proceso de aprendizaje nunca termina. Esto permite cuestionarnos si la asistencia permanente a las aulas o el contacto físico con profesores y otros estudiantes son pertinentes o si, por el contrario, se cuenta con la suficiente autonomía para asumir este proceso de manera independiente.

La autonomía influye de manera considerable en un proceso de educación a distancia, que conserva la dualidad enseñanza- aprendizaje. En este sentido, encontramos que el aprendizaje se convierte en un proceso continuo e independiente de la metodología empleada.

En la modalidad de educación a distancia, el aprendizaje no se centra en la presencia física de los docentes y estudiantes, ya no son ellos quienes se desplazan a las aulas, sino que la universidad llega a sus hogares, a sus sitios de trabajo o a sus centros de estudio. Entonces, se le exige un alto nivel de "aprendizaje autónomo" a los estudiantes, pues ellos mismos escogen sus sitios de trabajo, el tiempo necesario para desarrollar sus actividades, la metodología y sus estrategias de aprendizaje; “... los estudiantes aprenden por su propia cuenta y asimismo, tienen más responsabilidad y adquieren un cierto grado de autonomía en el aprendizaje (Rodríguez, 2009).

A pesar de que el estudiante es el verdadero responsable de su propio proceso de conocimiento y educación, la labor de los tutores es primordial en este proceso, ya que una de las esencias que tiene la educación a distancia es la mediación del docente. Se requiere de un aprendizaje independiente, pero ello no 
significa abandono, sino que es la representación directa de la autonomía orientada por los docentes-tutores.

Las habilidades con las que cuenta -o irá desarrollando- el estudiante es otro aspecto que actúa en este proceso de formación educativa, una de esas habilidades es la comprensión lectora.

Existen dos tipos de metodologías de educación, una es la modalidad presencial y la otra es a distancia. En la primera, las formas de interacción y de transmisión de conocimientos se basa en las clases magistrales, que implica el diálogo entre docentes y estudiantes o, en su defecto, el discurso del docente. Estos encuentros se presentan en un tiempo y lugar específicos.

En la segunda, las interacciones entre los actores del proceso de educación son diferentes; requieren de otros vehículos, ya que la mayor parte -o la totalidaddel proceso se lleva a cabo en ausencia de clases magistrales, docentes y estudiantes en espacios físicos (Rodríguez, 2009).

El material en el que se basan los estudiantes de educación a distancia sustituye la presencia física del profesor. La totalidad de dicho material se encuentra en documentos escritos y los estudiantes deben estudiarlos por su cuenta, para ello es indispensable dominar el proceso lector, así como contar con buenas competencias de comprensión lectora (Rodríguez, 2009), dado que "en educación a distancia la lectura se constituye en el proceso básico del acto académico, porque es, en esta habilidad donde radica la posibilidad de interpretar y comprender el texto escrito (editado o digitalizado)..."(Rodríguez, 2009).

En este artículo no es posible profundizar en todos los factores que influyen en un proceso exitoso; por eso, se considera importante finalizar con una breve mención de las competencias individuales y relacionales, necesarias para un buen proceso de educación a distancia, que cita Padilla (2008), con el fin de orientar a quien desee ahondar en este tema:

\section{Competencias individuales:}

- Motivación.

- Responsabilidad.

- Decisión.

- Iniciativa.

- Actitud.

- Liderazgo.

\section{Competencias relacionales:}

- Espíritu de equipo y colaboración-sinergia-.

- Comunicación y relaciones interpersonales.

- Transmisión y difusión de la información.

- Capacidad para ordenar y estimular a los demás.

Todo lo anterior justifica la idea de que, en efecto, la educación a distancia logra reducir las distancias físicas, ya que podemos estar a unos cuantos pasos, o muy cerca de grandes y exitosos sistemas 
de educación. Lo que se debe tener claro es que este sistema requiere de ciertas características personales para que se afiance el proceso constante de aprender a aprender.

Para concluir, se reafirma que es posible alcanzar metas importantes en espacios de educación a distancia, si se deja de lado la distancia como una excusa, ya que la educación a distancia brinda la posibilidad de que la educación llegue a nuestros hogares, y evita que nosotros tengamos que desplazarnos a los establecimientos educativos.

\section{Referencias}

1. BADIA, A.; Barberà, E.y Monimó,J. (2001). La incógnita de la educación a distancia. Barcelona: Horsori.
2. Facundo, A. (2004). La virtualización en la perspectiva de la modernización de la educación superior: consideraciones pedagógicas. Poliantea: revista académica y cultural Fundación Politécnico Grancolombiano Institución Universitaria, Núm. 2.

3. Ferroni, E. y Velásquez, H. (2005). Educación a distancia para el salto académico (Parte I). Poliantea. Revista académica y cultural Fundación Politécnico Grancolombiano Institución Universitaria, Núm. 4.

4. Padilla, J. (2008). Globalización y educación superior: un reto en la formación del docente universitario. Colección Itinerario educativo, Núm. 4. 